PROCEEDINGS OF THE

AMERICAN MATHEMATICAL SOCIETY

Volume 135, Number 12, December 2007, Pages 3995-4007

S 0002-9939(07)08922-8

Article electronically published on September 7, 2007

\title{
ON THE SKOROKHOD REPRESENTATION THEOREM
}

\author{
JEAN CORTISSOZ \\ (Communicated by Richard C. Bradley)
}

\begin{abstract}
In this paper we present a variant of the well-known Skorokhod Representation Theorem. First we prove, given $S$ a Polish Space, that to a given continuous path $\alpha$ in the space of probability measures on $S$, we can associate a continuous path in the space of $S$-valued random variables on a nonatomic probability space (endowed with the topology of the convergence in probability). We call this associated path a lifting of $\alpha$. An interesting feature of our result is that we can fix the endpoints of the lifting of $\alpha$, as long as their distributions correspond to the respective endpoints of $\alpha$. Finally, we also discuss and prove an $n$-dimensional generalization of this result.
\end{abstract}

\section{INTRODUCTION}

Let $(S, d)$ be a complete separable metric space and $(\Omega, \mathcal{F}, P)$ be a complete nonatomic probability space (here $\mathcal{F}$ denotes the $\sigma$-algebra where $P$ is defined), $\mathcal{P}(S)$ the space of probability measures on $S$, and $L^{0}(\Omega, S)$ the space of $S$-valued random variables. The Skorokhod Representation Theorem states the following.

Theorem 1. Suppose $P_{n}, n=1,2, \ldots$ and $P$ are probability measures on $S$ (endowed with its Borel $\sigma$-algebra) such that $P_{n} \Rightarrow P$ (see Section 2.2, Definition 2.5). Then there is a probability space $(\Omega, \mathcal{F}, P)$ on which are defined $S$-valued random variables $X_{n}, n=1,2, \ldots$ and $X$ with distributions $P_{n}$ and $P$, respectively, such that $\lim _{n \rightarrow \infty} X_{n}=X$ a.s.

A stronger result is presented in $[\mathrm{BD}$. The purpose of this paper is to prove results in the same spirit as Theorem 1 In the first place we prove the following.

Theorem 2. Let $\alpha:[0,1] \rightarrow \mathcal{P}(S)$ be a continuous function $(\mathcal{P}(S)$ endowed with the topology of the weak convergence; see Section 2.2). Let $X_{0}$ and $X_{1}$ be $S$-valued random variables defined on $(\Omega, \mathcal{F})$ such that law $\left(X_{0}\right)=\alpha(0)$ and law $\left(X_{1}\right)=$ $\alpha(1)$. There is $\hat{\alpha}:[0,1] \rightarrow L^{0}(\Omega, S)$ continuous $\left(L^{0}(\Omega, S)\right.$ endowed with the topology of the convergence in probability), such that $\hat{\alpha}(0)=X_{0}$ and $\hat{\alpha}(1)=X_{1}$ and $\operatorname{law}(\hat{\alpha})=\alpha$.

Then, using the techniques developed to show Theorem 2 plus a couple of ideas, we prove the following.

Received by the editors March 16, 2006 and, in revised form, July 6, 2006 and September 11, 2006.

2000 Mathematics Subject Classification. Primary 60B10.

(C)2007 American Mathematical Society Reverts to public domain 28 years from publication 
Theorem 3. Let $H:[0,1]^{n} \rightarrow \mathcal{P}(S)$ be a continuous function $(\mathcal{P}(S)$ endowed with the topology of the weak convergence). Let $X: \partial[0,1]^{n} \rightarrow L^{0}(\Omega, S)$ (D is equal to the boundary) be a continuous function such that law $(X)=\left.H\right|_{\partial[0,1]^{n}}$. There is $\hat{H}:[0,1]^{n} \rightarrow L^{0}(\Omega, S)$ continuous, such that $\left.\hat{H}\right|_{\partial[0,1]^{n}}=X$ and law $(\hat{H})=H$.

We must point out that the result in $\mathrm{BD}$ may seem stronger than the results described above. In a certain sense this is true, since given $\alpha:[0,1] \rightarrow \mathcal{P}(S)$ and a representation $\beta: \mathcal{P}(S) \times \Omega \longrightarrow S$ (using the definitions in [BD] and noticing that their $M(X)$ is our $\mathcal{P}(S)$ ) by defining $\hat{\alpha}(t)=\beta(\alpha(t), \cdot)$. With this definition, if $t_{n} \rightarrow t_{0}$, then $\hat{\alpha}\left(t_{n}\right) \rightarrow \hat{\alpha}\left(t_{0}\right)$ a.s., and a.s. convergence implies convergence in probability. However, in our results we are allowed to fix boundary values for the liftings (or representations). As a curious, yet interesting, consequence, Theorem 3 yields

Corollary 1. All the homotopy groups of $L^{0}(\Omega, S)$ (endowed with the topology of convergence in probability) are trivial.

This paper is arranged as follows: in Section 2 we collect some basic theory as a quick reference for the convenience of the reader. In Section 3 we prepare some lemmas we use in the proof of Theorem 2, which we finally prove in Section 4. The proof of Theorem 3 is at the end of Section 4 .

\section{Preliminaries}

In this section we collect some important and well-known definitions and facts for the reader's convenience. We recommend Chapter 3 of $[\mathrm{EK}]$ as a reference for this section.

\subsection{Convergence in probability.}

Definition 2.1. We say that a sequence $\left(X_{n}\right)_{n}$ converges in probability to $X$, and we denote it by $X_{n} \stackrel{P}{\rightarrow} X$, if for every $\epsilon>0$ we have

$$
\lim _{n \rightarrow \infty} P\left\{\omega: d\left(X_{n}(\omega), X(\omega)\right) \geq \epsilon\right\}=0 .
$$

Definition 2.2. Given $X$ and $Y$ random variables, define

$$
\rho(X, Y)=\inf \{\epsilon>0: P\{\omega: d(X(\omega), Y(\omega)) \geq \epsilon\} \leq \epsilon\} .
$$

Theorem 2.1. If $\rho$ is a metric on $L^{0}(\Omega, S)$ and is given a sequence $\left(X_{n}\right)_{n}$ of random variables and a random variable $X$, then

$$
\lim _{n \rightarrow \infty} \rho\left(X_{n}, X\right)=0 \quad \text { if and only if } \quad X_{n} \stackrel{P}{\rightarrow} X .
$$

\subsection{The space $\mathcal{P}(S)$ and the function law.}

Definition 2.3. Let $\mathcal{B}(S)$ be the collection of Borel subsets of $S$. Then we define

$$
\mathcal{P}(S)=\{\mu: \mu \text { is a probability measure on } \mathcal{B}(S)\} .
$$

Definition 2.4. Given $\mu \in \mathcal{P}(S)$ and $A \in \mathcal{B}(S)$ we say that $A$ is a set of $\mu$ continuity if $\mu(\partial A)=0(\partial A$ is the topological boundary of $A)$.

A sequence $\left(\mu_{n}\right)_{n}$ in $\mathcal{P}(S)$ converges weakly to $\mu$, and we denote it by $\mu_{n} \Rightarrow \mu$, if for every set of $\mu$-continuity $A, \mu_{n}(A) \rightarrow \mu(A)$ as $n \rightarrow \infty$. 
Definition 2.5. Given $\mu, \nu \in \mathcal{P}(S)$ define

$$
q(\mu, \nu)=\inf \left\{\epsilon>0: \mu(A) \leq \nu\left(A^{\epsilon}\right)+\epsilon \text { for all } A \subset S \text { closed }\right\},
$$

where $A^{\epsilon}=\{x \in S: \exists y \in A$ such that $d(x, y)<\epsilon\}$.

Theorem 2.2. If $q$ defines a metric on $\mathcal{P}(S)$, and given a sequence $\left(\mu_{n}\right)_{n}$ and a probability measure $\mu$, then

$$
\lim _{n \rightarrow \infty} q\left(\mu_{n}, \mu\right)=0 \quad \text { if and only if } \mu_{n} \Rightarrow \mu .
$$

Definition 2.6. Given $X \in L^{0}(\Omega, S)$, the probability measure $\mu$ defined on $\mathcal{B}(S)$ by

$$
\mu(A)=P\left(X^{-1}(A)\right)
$$

is called the distribution or law of $X$.

\section{A CRUCial LEMma}

Theorem 3.1. Let $(S, d)$ be separable, and let $P, Q \in \mathcal{P}(S)$. Define $\mathcal{M}(P, Q)$ to be the set of all $\mu \in \mathcal{P}(S \times S)$ with marginals $P$ and $Q$. Then

$$
q(P, Q)=\inf _{\mu \in \mathcal{M}(P, Q)} \inf \{\epsilon>0: \mu\{(x, y): d(x, y) \geq \epsilon\} \leq \epsilon\} .
$$

Proof. See [EK] (Chapter 3, Theorem 1.2) or [S] (Corollary to Theorem 10).

As a corollary we get

\section{Corollary 3.1.}

$$
q(P, Q)=\inf \{\rho(X, Y): \operatorname{law}(X)=P \quad \text { and } \quad \text { law }(Y)=Q\} .
$$

Proof. Since $\Omega$ is nonatomic, given $\mu \in \mathcal{M}(P, Q)$ there is a random variable $W \in$ $L^{0}(\Omega, S \times S)$ such that $l a w(W)=\mu$. But by its very definition $W=(X, Y)$, where $X, Y \in L^{0}(\Omega, S)$ and law $(X)=P$ and $\operatorname{law}(Y)=Q$. Then all we have to notice is that

$$
\rho(X, Y)=\inf \{\epsilon>0: P\{\omega: d(X(\omega), Y(\omega)) \geq \epsilon\} \leq \epsilon\} .
$$

Remark 1. Corollary 3.1 has as a consequence that the function

$$
\text { law }:\left(L^{0}(\Omega, S), \rho\right) \longrightarrow(\mathcal{P}(S), q)
$$

is continuous (indeed, it shows that it is distance decreasing).

We will use the following "working" definitions.

Definition 3.1. $\delta_{a}$ is the measure defined by

$$
\delta_{a}(V)= \begin{cases}1 & \text { if } a \in V \\ 0 & \text { otherwise }\end{cases}
$$

We say that $\mu$ is finitely supported if it can be written as

$$
\mu=\sum_{i=1}^{n} c_{i} \delta_{a_{i}}, \quad c_{i} \geq 0 .
$$

Definition 3.2. Let $\left(A_{k}\right)_{k=1, \ldots, n}$ be a partition of $\Omega$. Define the simple $S$-valued random variable $X=\sum_{i=1}^{n} a_{i} \chi_{A_{i}}$ as

$$
X(\omega)=a_{i} \quad \text { iff } \quad \omega \in A_{i} .
$$

Corollary 3.1 is used to prove 
Lemma 3.1. Let $\mu$ and $\nu$ be finitely supported measures, and let $\epsilon>0$ be such that

$$
q(\mu, \nu)<\epsilon \text {. }
$$

Then, given $X$ a random variable such that law $(X)=\mu$, there exists $Y$ such that law $(Y)=\nu$ and $\rho(X, Y)<\epsilon$.

Proof. Since $\mu$ and $\nu$ have finite support, by Theorem 3.1 we can find

$$
X^{\prime}=\sum_{j=1}^{m} a_{j} \chi_{A_{j}^{\prime}} \quad \text { and } \quad Y^{\prime}=\sum_{j=1}^{m} a_{j} \chi_{B_{j}^{\prime}},
$$

where $\left\{a_{1}, \ldots, a_{m}\right\}$ is the union of the supports of $\mu$ and $\nu$, and so that $l a w\left(X^{\prime}\right)=\mu$, law $\left(Y^{\prime}\right)=\nu$ and $\rho\left(X^{\prime}, Y^{\prime}\right)<\epsilon$.

Write $X=\sum_{j=1}^{m} a_{j} \chi_{A_{j}}$. Since $\Omega$ is nonatomic we can find measurable sets $B_{1}, B_{2}, \ldots, B_{m}$ such that

$$
P\left(A_{i} \cap B_{j}\right)=P\left(A_{i}^{\prime} \cap B_{j}^{\prime}\right) \quad \text { for all } \quad i, j=1,2, \ldots, m .
$$

It is then clear that $Y=\sum_{j=1}^{m} a_{j} \chi_{B_{j}}$ satisfies $\rho(X, Y)<\epsilon$.

Finally we have the following crucial lemma.

Lemma 3.2. Let $\epsilon>0$ be given and assume $q($ law $(X)$, law $(Y))<\epsilon$. Then there is $Y^{\prime}$ such that law $\left(Y^{\prime}\right)=\operatorname{law}(Y)$ and $\rho\left(X, Y^{\prime}\right)<\epsilon$.

Proof. We proceed by induction on the complexity of random variables. The case when $X$ and $Y$ are simple is contained in Lemma 3.1 .

Now assume that $X$ is arbitrary and $Y$ is simple. Let $\delta>0$ be such that

$$
q(\operatorname{law}(X), \operatorname{law}(Y))<\epsilon-\delta .
$$

Since the set of simple random variables is dense in $L^{0}(\Omega, S)$, and the function law is continuous (see the Remark after Corollary [3.1), we can choose $X^{\prime}$ to be a simple random variable such that $\rho\left(X, X^{\prime}\right)<\delta$, and $q\left(\operatorname{law}\left(X^{\prime}\right)\right.$, law $\left.(Y)\right)<\epsilon-\delta$. By the induction hypothesis, we can find $Y^{\prime}$ such that $\operatorname{law}\left(Y^{\prime}\right)=\operatorname{law}(Y)$ and $\rho\left(X^{\prime}, Y^{\prime}\right)<\epsilon-\delta$. Hence we have

$$
\rho\left(X, Y^{\prime}\right) \leq \rho\left(X, X^{\prime}\right)+\rho\left(X^{\prime}, Y^{\prime}\right)<\delta+\epsilon-\delta=\epsilon .
$$

Finally, let $X$ and $Y$ be arbitrary random variables and let $\delta>0$ be such that

$$
q(\operatorname{law}(X), \operatorname{law}(Y))+2 \delta<\epsilon .
$$

Choose a sequence $\left(Y_{n}\right)_{n}$ of simple random variables converging to $Y$ and such that

$$
\rho\left(Y_{n}, Y_{n+1}\right)<\frac{1}{2^{n+1}} \quad \text { and } \quad q\left(\operatorname{law}\left(Y_{n}\right), \operatorname{law}(Y)\right)<\delta,
$$

and let $N$ be such that $\frac{1}{2^{N}}<\delta$ and also $\rho\left(Y_{N}, Y\right)<\delta$. We construct a new sequence $\left(Y_{j}^{\prime}\right)_{j=N, N+1, N+2, \ldots}$ as follows:

If $j=N$, choose $Y_{N}^{\prime}$ such that $\operatorname{law}\left(Y_{N}^{\prime}\right)=\operatorname{law}\left(Y_{N}\right)$ and

$$
\rho\left(Y_{N}^{\prime}, X\right)<q(\operatorname{law}(X), \operatorname{law}(Y))+\delta .
$$

This can be done because

$$
\begin{aligned}
q\left(\operatorname{law}\left(Y_{N}\right), \operatorname{law}(X)\right) & \leq q\left(\operatorname{law}\left(Y_{N}\right), \operatorname{law}(Y)\right)+q(\operatorname{law}(Y), \operatorname{law}(X)) \\
< & q(\operatorname{law}(Y), \operatorname{law}(X))+\delta .
\end{aligned}
$$


Once we have chosen $Y_{j}^{\prime}$ for $j=N+1, \ldots, N+M$, we pick $Y_{N+M+1}^{\prime}$ such that

$$
\operatorname{law}\left(Y_{N+M+1}\right)=\operatorname{law}\left(Y_{N+M+1}^{\prime}\right) \quad \text { and } \rho\left(Y_{N+M+1}^{\prime}, Y_{N+M}^{\prime}\right)<\frac{1}{2^{N+M+1}},
$$

which can be justified because

$$
q\left(\operatorname{law}\left(Y_{N+M+1}\right), \operatorname{law}\left(Y_{N+M}^{\prime}\right)\right)=q\left(\operatorname{law}\left(Y_{N+M+1}\right), \operatorname{law}\left(Y_{N+M}\right)\right)<\frac{1}{2^{N+M+1}} .
$$

By construction, the sequence $\left(Y_{j}^{\prime}\right)_{j}$ is convergent, and for its limit $Y^{\prime}$ it follows that $\operatorname{law}\left(Y^{\prime}\right)=\operatorname{law}(Y)$, and also

$$
\begin{aligned}
\rho\left(X, Y^{\prime}\right) & \leq \rho\left(X, Y_{N}^{\prime}\right)+\sum_{j=1} \frac{\delta}{2^{j}} \\
& <q(\operatorname{law}(X), \operatorname{law}(Y))+\delta+\delta<\epsilon .
\end{aligned}
$$

\section{Representation theorems}

4.1. Liftings. First we give a definition we learned from Ramiro de La Vega ([V]).

Definition 4.1. A family of measurable sets $\left(A_{t}\right)_{t \in[0, \delta]}$ is a $[0, \delta]$-family if it satisfies: (i) $A_{s} \subset A_{t}$ whenever $s \leq t$, and (ii) $P\left(A_{t}\right)=t$.

The following lemma, which we also learned from de La Vega, is what makes $[0, \delta]$-families a useful tool.

Lemma 4.1. Let $(\Omega, \mathcal{F}, P)$ be a complete nonatomic probability space, and let $A \in \mathcal{F}$ and let $\delta=P(A)$. Then there is a $[0, \delta]$-family $\left(A_{t}\right)_{t \in[0, \delta]}$ such that $A_{t} \subset A$ for all $t$.

Proof. Pick an ordering of $\mathbf{Q} \cap[0,1]\left(\mathbf{Q}=\right.$ the rational numbers), say $q_{1}, q_{2}, q_{3}, \ldots$ . Since $\Omega$ is nonatomic we can inductively construct $E_{q_{1}}, E_{q_{2}}, E_{q_{3}}, \ldots$ such that

$$
P\left(E_{q}\right)=q, \quad \text { and } \quad E_{q} \subset E_{r} \quad \text { if } \quad q \leq r .
$$

Finally for $x \in[0,1] \backslash \mathbf{Q}$, define $E_{x}=\bigcup_{q<x} E_{q}$.

Using $[0, \delta]$-families we can introduce the notion of a segment joining two simple random variables.

Definition 4.2. Let $X=\sum_{j=1}^{m} a_{j} \chi_{A_{j}}$ and $Y=\sum_{j=1}^{m} a_{j} \chi_{B_{j}}$ be two simple random variables. Define $E_{i j}=A_{i} \cap B_{j}$ and $e_{i j}=P\left(E_{i j}\right)$, and let $\left(\left[E_{i j}\right]_{t}\right)$ be a $\left[0, e_{i j}\right]$ family of $E_{i j}$. A segment $\alpha_{X, Y}:[a, b] \rightarrow L^{0}(\Omega, S)$ joining $X$ to $Y$ is defined as

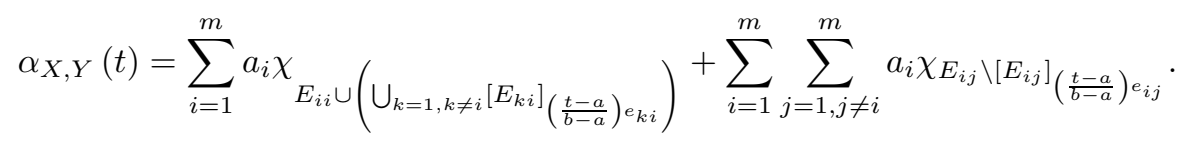

We describe some important properties of these segments.

Proposition 4.1. $\hat{\alpha}:=\alpha_{X, Y}$ thus defined is a continuous function with $\alpha_{X, Y}(a)=$ $X$ and $\alpha_{X, Y}(b)=Y$. Moreover,

$$
\alpha(t):=\operatorname{law}(\hat{\alpha}(t))=\left(\frac{b-t}{b-a}\right) \operatorname{law}(X)+\left(\frac{t-a}{b-a}\right) \operatorname{law}(Y) .
$$


Proof. First we show that $\alpha_{X, Y}$ is continuous. It is an immediate consequence of the following inequality. Let $\epsilon>0$ be given and $s \leq t$. Then we have

$$
\begin{aligned}
P\{\omega: d(\hat{\alpha}(t), \hat{\alpha}(s)) \geq \epsilon\} & \leq \sum_{\left\{(i, k): d\left(a_{i}, a_{k}\right) \geq \epsilon\right\}} P\left(\left[E_{i k}\right]_{(t-s) e_{i k}}\right) \\
& \leq(t-s) \sum_{\ldots} e_{i k} \leq t-s .
\end{aligned}
$$

Let us show that $\operatorname{law}(\hat{\alpha})=\alpha$. To make things easier, we will assume $a=0$ and $b=1$. Then all we must show is that the coefficient of $\delta_{a_{i}}$ in law $(\hat{\alpha})$ is $(1-t) P\left(A_{i}\right)+t P\left(B_{i}\right)$. Let's fix $i=1$. Then the sought coefficient is given by

$$
\begin{aligned}
P & \left(E_{11}\right)+\sum_{j=2}^{m} P\left(\left[E_{j 1}\right]_{t e_{j 1}}\right)+\sum_{j=2}^{m} P\left(E_{1 j} \backslash\left[E_{1 j}\right]_{t e_{1 j}}\right) \\
& =e_{11}+\sum_{j=2}^{m} t e_{j 1}+\sum_{j=2}^{m}\left(e_{1 j}-t e_{1 j}\right) \\
& =P\left(A_{1}\right)+t\left(P\left(B_{1}\right)-e_{11}\right)-t \sum_{j=2}^{m} e_{1 j} \\
& =P\left(A_{1}\right)+t P\left(B_{1}\right)-t \sum_{j=1}^{m} e_{1 j}=P\left(A_{1}\right)+t P\left(B_{1}\right)-t P\left(A_{1}\right) .
\end{aligned}
$$

To approximate continuous maps from $[0,1]$ to $\mathcal{P}(S)$, we will use a special family of paths we call "polygonals".

Definition 4.3 (Polygonals). We call $\beta:[0,1] \longrightarrow \mathcal{P}(S)$ a polygonal with vertices at $\mu_{0}, \ldots, \mu_{n+1}$ if there is a partition $0=t_{0}, t_{1}, \ldots, t_{n}, t_{n+1}=1$ of $[0,1]$ such that $\beta$ restricted to $\left[t_{i}, t_{i+1}\right]$ is given by

$$
\beta(t)=\left(\frac{t_{i+1}-t}{t_{i+1}-t_{i}}\right) \mu_{i}+\left(\frac{t-t_{i}}{t_{i+1}-t_{i}}\right) \mu_{i+1} .
$$

We call the function $\left.\beta\right|_{\left[t_{i}, t_{i+1}\right]}$, defined as above, the segment (with domain $\left[t_{i}, t_{i+1}\right]$ ) joining $\mu_{i}$ to $\mu_{i+1}$.

An easy consequence of Proposition 4.1 is the following fact about polygonals.

Proposition 4.2. Let $\alpha:[0,1] \longrightarrow \mathcal{P}(S)$ be a polygonal with vertices at measures of finite support. Given $X_{0}$ and $X_{1}$ such that law $\left(X_{0}\right)=\alpha(0)$ and law $\left(X_{1}\right)=$ $\alpha(1)$, there is a lifting $\hat{\alpha}:[0,1] \longrightarrow L^{0}(\Omega, S)$ (i.e., law $\left.(\hat{\alpha}(t))=\alpha(t)\right)$, such that $\hat{\alpha}(0)=X_{0}$ and $\hat{\alpha}(1)=X_{1}$.

As was announced, polygonals are dense in the space of continuous maps from the unit interval to $\mathcal{P}(S)$. Before we write and prove the exact statement of this fact we will need the following observation.

Lemma 4.2. Let $\mu, \nu \in \mathcal{P}(S)$. For $t \in[0,1]$ we have

$$
q(\nu, t \mu+(1-t) \nu) \leq q(\nu, \mu) .
$$

Proof. Let $\epsilon>0$ be such that $\mu(A) \leq \nu\left(A^{\epsilon}\right)+\epsilon$ for all $A \subset S$ closed. Then we have

$$
\begin{aligned}
t \mu(A)+(1-t) \nu(A) & \leq t \nu\left(A^{\epsilon}\right)+t \epsilon+(1-t) \nu\left(A^{\epsilon}\right)+(1-t) \epsilon \\
& =\nu\left(A^{\epsilon}\right)+\epsilon,
\end{aligned}
$$

and from this the statement of the lemma follows. 
Now we are ready to state and prove the following density property of polygonals.

Lemma 4.3. Given $\alpha:[0,1] \longrightarrow \mathcal{P}(S)$ a continuous function and $\epsilon>0$, there is a polygonal $\beta$ with vertices at measures of finite support such that

$$
\sup _{t \in[0,1]} q(\alpha(t), \beta(t))<\epsilon
$$

Proof. Let $\epsilon>0$ be given. By the uniform continuity of $\alpha$, we can find $\delta>0$ such that whenever $|s-t|<\delta$ we have $q(\alpha(t), \alpha(s))<\frac{\epsilon}{5}$. Let $N>0$ be big enough so that $\frac{1}{N}<\delta$, and define a partition of the interval $[0,1]$ by $t_{i}=\frac{i}{N} i=0,1, \ldots, N$. For each $i$ pick a finitely supported measure $\mu_{i}$ such that $q\left(\mu_{i}, \alpha\left(t_{i}\right)\right) \leq \frac{\epsilon}{5}$. Let $\beta$ be the polygonal defined by the segments $\beta:\left[t_{i}, t_{i+1}\right] \longrightarrow \mathcal{P}(S)$ with endpoints $\mu_{i}$ and $\mu_{i+1}$. For $t \in\left[t_{i}, t_{i+1}\right]$ we have

$$
\begin{aligned}
q(\alpha(t), \beta(t)) \leq & q\left(\alpha(t), \alpha\left(t_{i}\right)\right)+q\left(\alpha\left(t_{i}\right), \mu_{i}\right)+q\left(\mu_{i}, \beta(t)\right) \\
\text { (by Lemma 4.2) } & \leq q\left(\alpha(t), \alpha\left(t_{i}\right)\right)+q\left(\alpha\left(t_{i}\right), \mu_{i}\right)+q\left(\mu_{i}, \mu_{i+1}\right) \\
\leq & q\left(\alpha(t), \alpha\left(t_{i}\right)\right)+q\left(\alpha\left(t_{i}\right), \mu_{i}\right)+q\left(\mu_{i}, \alpha\left(t_{i}\right)\right) \\
& +q\left(\alpha\left(t_{i}\right), \alpha\left(t_{i+1}\right)\right)+q\left(\alpha\left(t_{i+1}\right), \mu_{i+1}\right) \\
< & \frac{\epsilon}{5}+\frac{\epsilon}{5}+\frac{\epsilon}{5}+\frac{\epsilon}{5}+\frac{\epsilon}{5}=\epsilon .
\end{aligned}
$$

4.2. Proof of Theorem 2. We are almost ready to prove Theorem 2 of this paper. The following fact will be important in its proof.

Lemma 4.4. Let $\alpha:[0,1] \rightarrow \mathcal{P}(S)$ be continuous and let $\epsilon>0$ be given. Let $\beta$ be an arbitrary polygonal with vertices at measures of finite support and such that

$$
\sup _{t \in[0,1]} q(\alpha(t), \beta(t))<\epsilon
$$

Then, given any continuous lifting $\hat{\alpha}$ of $\alpha$, there is a lifting $\hat{\beta}$ of $\beta$ such that

$$
\sup _{t \in[0,1]} \rho(\hat{\alpha}(t), \hat{\beta}(t))<5 \epsilon .
$$

For the proof of this lemma we need the following observation.

Lemma 4.5. Let $X_{\mu}=\sum_{j=1}^{m} a_{j} \chi_{A_{j}}$ and $X_{\nu}=\sum_{j=1}^{m} a_{j} \chi_{B_{j}}$ be such that law $\left(X_{\mu}\right)$ $=\mu$ and law $\left(X_{\nu}\right)=\nu$ are finitely supported measures. Let $\hat{\alpha}:=\alpha_{X_{\mu}, X_{\nu}}$ be as in Definition 4.2. Then we have $\rho\left(X_{\mu}, \hat{\alpha}(t)\right) \leq \rho\left(X_{\mu}, X_{\nu}\right)$.

Proof. (We use the notation of Definition 4.2]) Given $\epsilon>0$ we have

$$
\begin{aligned}
P\left\{\omega: d\left(X_{\mu}(\omega), X_{\nu}(\omega)\right) \geq \epsilon\right\} & =\sum_{\left\{(i, j): d\left(a_{i}, a_{j}\right) \geq \epsilon\right\}} P\left(E_{i j}\right) \\
& \geq \sum_{\left\{(i, j): d\left(a_{i}, a_{j}\right) \geq \epsilon\right\}} P\left(\left[E_{i j}\right]_{t e_{i j}}\right) \\
& =P\left\{\omega: d\left(X_{\mu}(\omega), \hat{\alpha}(t)\right) \geq \epsilon\right\} .
\end{aligned}
$$

The conclusion of the lemma follows.

Proof of Lemma 4.4. Let $\hat{\alpha}$ be a continuous lifting of $\alpha$. Take a partition $0=t_{0}<$ $t_{1}<\cdots<t_{n+1}=1$ of the unit interval, in such a way that

$$
\rho\left(X_{i}, X_{i+1}\right)<\epsilon \text { where } X_{i}=\hat{\alpha}\left(t_{i}\right) .
$$

Choose $Y_{i}$ for $i=0,1, \ldots, n+1$ so that $\operatorname{law}\left(Y_{i}\right)=\beta\left(t_{i}\right)$ and $\rho\left(X_{i}, Y_{i}\right)<\epsilon$. Then we have

$$
\rho\left(Y_{i}, Y_{i+1}\right) \leq \rho\left(Y_{i}, X_{i}\right)+\rho\left(X_{i}, X_{i+1}\right)+\rho\left(X_{i+1}, Y_{i+1}\right)<3 \epsilon .
$$


Construct a lifting $\hat{\beta}$ of $\beta$, such that $\hat{\beta}$ restricted to the segment $\left[t_{i}, t_{i+1}\right]$ is a lifting of $\beta:\left[t_{i}, t_{i+1}\right] \longrightarrow \mathcal{P}(S)$ with $\hat{\beta}\left(t_{i}\right)=Y_{i}$ as given by Definition 4.2 Then $\hat{\beta}$ is continuous and for $t_{i} \leq t<t_{i+1}$ we have

$$
\begin{aligned}
\rho(\hat{\alpha}(t), \hat{\beta}(t)) & \leq \rho\left(\hat{\alpha}(t), X_{i}\right)+\rho\left(X_{i}, Y_{i}\right)+\rho\left(Y_{i}, \hat{\beta}(t)\right) \\
\text { (by Lemma 4.5) } & \leq \rho\left(\hat{\alpha}(t), X_{i}\right)+\rho\left(X_{i}, Y_{i}\right)+\rho\left(Y_{i}, Y_{i+1}\right) \\
& <\epsilon+\epsilon+3 \epsilon=5 \epsilon .
\end{aligned}
$$

Remark 2. Notice that from the proof of Lemma 4.4, we can prescribe the values of $\hat{\beta}(i)(i=0,1)$ as long as they are simple random variables and $\rho(\hat{\beta}(i), \hat{\alpha}(i))<\epsilon$.

Proof of Theorem 2. Take a sequence of polygonals $\left(\alpha_{n}\right)_{n}$ with vertices at measures of finite support and such that

$$
\alpha_{n} \rightarrow \alpha \quad \text { and } \sup _{t \in[0,1]} q\left(\alpha_{n}(t), \alpha_{n+1}(t)\right)<\frac{1}{5^{n+1}}
$$

By Proposition 4.2 and Lemma 4.4, we can lift this sequence to a sequence $\left(\hat{\alpha}_{n}\right)_{n}$ so that $\hat{\alpha}_{n}(0) \rightarrow X_{0}, \hat{\alpha}_{n}(1) \rightarrow X_{1}$ and $\sup _{t \in[0,1]} \rho\left(\hat{\alpha}_{n}(t), \hat{\alpha}_{n+1}(t)\right)<\frac{1}{5^{n}}$.

It is clear by construction that $\left(\hat{\alpha}_{n}\right)_{n}$ is a convergent sequence. Let $\hat{\alpha}$ be its limit. Then, since the convergence is uniform, $\hat{\alpha}$ is continuous, and because law is a continuous function, law $(\hat{\alpha}(t))=\alpha(t)$. This finishes the proof.

4.3. On Theorem [3. In order to give a proof of Theorem 3] we will introduce a few definitions.

Definition 4.4. The 1 -face with vertices $\mu_{a_{1}}=\alpha^{1}\left(a_{1}\right)$ and $\mu_{b_{1}}=\alpha^{1}\left(b_{1}\right)$ is the segment $\alpha^{1}:\left[a_{1}, b_{1}\right] \longrightarrow \mathcal{P}(S)$ joining $\mu_{a_{1}}$ to $\mu_{b_{1}}$ (see Definition 4.3). To construct the $n$-face $\alpha^{n}: \Pi_{k=1}^{n}\left[a_{k}, b_{k}\right] \longrightarrow \mathcal{P}(S)$ with vertices $\mu_{i_{1}, i_{2}, \ldots, i_{n-1}, i_{n}}$ $\left(i_{k}=a_{k}, b_{k}, k=1, \ldots n\right)$ at measures of finite support we proceed as follows. Define $\alpha^{n}\left(i_{1}, \ldots, i_{n-1}, t_{n}\right):\left[a_{n}, b_{n}\right] \longrightarrow \mathcal{P}(S)$ as the segment joining $\mu_{i_{1}, \ldots, i_{n-1}, a_{n}}$ to $\mu_{i_{1}, \ldots, i_{n-1}, b_{n}}$. Finally, for $t_{n}$ fixed, define $\alpha_{t_{n}}^{n}: \Pi_{k=1}^{n-1}\left[a_{k}, b_{k}\right] \longrightarrow \mathcal{P}(S)$ as the $(n-1)$-face with vertices $\alpha_{t_{n}}^{n}\left(i_{1}, \ldots, i_{n-1}\right)=\alpha^{n}\left(i_{1}, \ldots, i_{n-1}, t_{n}\right)$.

It is not difficult to convince oneself that $n$-faces are continuous functions: one can even write explicit formulae for them. For instance, $\alpha:[0,1]^{2} \longrightarrow \mathcal{P}(S)$ defined by $\alpha(t, s)=(1-s)\left((1-t) \mu_{0,0}+t \mu_{1,0}\right)+s\left((1-t) \mu_{0,1}+t \mu_{1,1}\right)$ is the 2 -face with vertices at $\alpha(i, j)=\mu_{i, j}, i, j=0,1$. We can also give the following more formal argument: the continuity of $n$-faces is a consequence of Proposition 4.3 (see later) and the fact that the function law is continuous.

Definition 4.5. We call a continuous function $H^{n}:[0,1]^{n} \longrightarrow \mathcal{P}(S)$ an $n$ polyhedral if there is a partition $0=t_{0}<t_{1}<\cdots<t_{n-1}<t_{n}=1$ such that $H^{n}$ restricted to $\Pi_{k=1}^{n}\left[t_{i_{k}}, t_{i_{k}+1}\right]$ is an $n$-face. We call the values $H^{n}\left(t_{k_{1}}, \ldots, t_{k_{n}}\right)$, $k, l=0, \ldots, n$, vertices of the $n$-polyhedral.

We must observe that $n$-polyhedrals are dense in the space of continuous functions from $[0,1]^{n}$ to $\mathcal{P}(S)$, the proof of this fact being a straightforward generalization of the proof of Lemma 4.3. Indeed, let $\epsilon>0$, and given a continuous $H_{1}:[0,1]^{n} \longrightarrow \mathcal{P}(S)$ pick $\delta>0$ such that if $|\mathbf{t}-\mathbf{s}|<\delta$, then $\left|H_{1}(\mathbf{t})-H_{1}(\mathbf{s})\right|<\epsilon$. Choose a partition $0=t_{0}<t_{1}<\cdots<t_{n-1}<t_{n}=1$ such that $t_{i+1}-t_{i}<\delta$. Let $\mu_{i_{1}, \ldots, i_{n}}$ be measures of finite support such that $q\left(\mu_{i_{1}, \ldots, i_{n}}, H_{1}\left(t_{i_{1}}, \ldots, t_{i_{n}}\right)\right)<\epsilon$. Construct the $n$-polyhedral of vertices $\mu_{i_{1}, \ldots, i_{n}}$ by constructing the $n$-faces $\alpha_{k_{1}, \ldots, k_{n}}$ : 
$\Pi_{j=1}^{n}\left[t_{k_{j}}, t_{k_{j}+1}\right] \longrightarrow \mathcal{P}(S)$ with the obvious vertices. Given $\mathbf{t} \in \Pi_{j=1}^{n}\left[t_{k_{j}}, t_{k_{j}+1}\right]$, then repeated applications of Lemma 4.2 and the triangle inequality show that $q\left(\alpha_{k_{1}, \ldots, k_{n}}(\mathbf{t}), \mu_{k_{1}, \ldots, k_{n}}\right)<n \epsilon$. This together with another use of the triangle inequality shows that $\sup _{\mathbf{t} \in[0,1]^{n}} q\left(H_{1}(\mathbf{t}), H_{2}(\mathbf{t})\right)<(n+2) \epsilon$.

We will need a canonical way of defining $[0, \eta]$-families of measurable subsets of $\Omega$. This is the reason for introducing the following.

Definition 4.6. Let $A, B$ be such that $B \subseteq A$. Let $\left([A]_{t}\right)_{t \in[0, \alpha]}$ be a $[0, P(A)]$ family of $A$. We define the induced $[0, P(B)]$-family on $B$ as follows:

$$
[B]_{s}=B \cap[A]_{\sup \left\{t: P\left(B \cap\left[A_{t}\right]\right)=s\right\}} .
$$

One of the most important properties of induced families is that they satisfy a continuity property; in other words, if two measurable sets are close, so are their induced families.

Lemma 4.6 (Continuity of induced families). If $P(A \Delta B)<\epsilon$, then

$$
P\left([A]_{t P(A)} \Delta[B]_{t P(B)}\right) \leq 2 \epsilon+t|P(A)-P(B)| .
$$

Proof. First we have

(1)

$$
\begin{aligned}
{[A]_{t P(A)} \Delta[B]_{t P(B)} } & \subseteq\left([A]_{t P(A)} \backslash B\right) \\
& \cup\left([B]_{t P(B)} \backslash A\right) \cup\left(\left([A]_{t P(A)} \cap B\right) \Delta\left([B]_{t P(B)} \cap A\right)\right) .
\end{aligned}
$$

Notice that by their definition, either $[A]_{t P(A)} \cap B \subseteq[B]_{t P(B)} \cap A$ or $[B]_{t P(B)} \cap A \subseteq$ $[A]_{t P(A)} \cap B$. In the first case, by (1) and the hypothesis,

$$
P\left([A]_{t P(A)} \Delta[B]_{t P(B)}\right) \leq \epsilon+t P(B)-(t P(A)-\epsilon) .
$$

The second case is similar. This shows the lemma.

From now on we fix a background $[0,1]$-family of $\Omega$, and all the $[0, \eta]$-families of measurable subsets of $\Omega$ considered will be induced by this background family. We are ready to construct liftings of $n$-faces.

Definition 4.7. The 1-extension with vertices at the simple random variables $X_{a_{1}}$ and $X_{b_{1}}$ is the segment $\hat{\alpha}^{1}:\left[a_{1}, b_{1}\right] \longrightarrow L^{0}(\Omega, S)$ joining $X_{a_{1}}$ to $X_{b_{1}}$ (remember we have fixed once and for all the choice of induced families). To construct the $n$-extension $\hat{\alpha}^{n}: \Pi_{k=1}^{n}\left[a_{k}, b_{k}\right] \longrightarrow L^{0}(\Omega, S)$ with vertices $X_{i_{1}, i_{2}, \ldots, i_{n-1}, i_{n}}$ $\left(i_{k}=a_{k}, b_{k}, k=1, \ldots, n\right)$ simple random variables we proceed as follows. Define $\hat{\alpha}^{n}\left(i_{1}, \ldots, i_{n-1}, t_{n}\right):\left[a_{n}, b_{n}\right] \longrightarrow L^{0}(\Omega, S)$ as the segment joining $X_{i_{1}, \ldots, i_{n-1}, a_{n}}$ to $X_{i_{1}, \ldots, i_{n-1}, b_{n}}$. Finally, given $t_{n}$ define $\hat{\alpha}_{t_{n}}^{n}: \Pi_{k=1}^{n-1}\left[a_{k}, b_{k}\right] \longrightarrow L^{0}(\Omega, S)$ as the $(n-1)$-extension with vertices $\hat{\alpha}_{t_{n}}^{n}\left(i_{1}, \ldots, i_{n-1}\right)=\hat{\alpha}^{n}\left(i_{1}, \ldots, i_{n-1}, t_{n}\right)$.

It is rather clear that given $\alpha^{n}: \prod_{k=1}^{n}\left[a_{k}, b_{k}\right] \longrightarrow \mathcal{P}(S)$, an $n$-face with vertices $\mu_{i_{1}, \ldots, i_{n}}\left(i_{k}=a_{k}\right.$ or $\left.b_{k}, k=1, \ldots, n\right)$, and simple random variables $X_{i_{1}, \ldots, i_{n}}$ such that law $\left(X_{i_{1}, \ldots, i_{n}}\right)=\mu_{i_{1}, \ldots, i_{n}}$, then there exists an $n$-extension $\hat{\alpha}: \prod_{k=1}^{n}\left[a_{k}, b_{k}\right] \longrightarrow$ $L^{0}(\Omega, S)$ with vertices $X_{i_{1}, \ldots, i_{n}}$ and such that $\operatorname{law}\left(\hat{\alpha}^{n}(t)\right)=\alpha^{n}(t)$.

We must show that $n$-extensions are continuous. Before we proceed, we prove the following basic fact. 
Lemma 4.7. Let $a_{1}, \ldots, a_{n} \in S$. There exist $\epsilon>0$ and $C>0$ such that for any $X_{i}=\sum_{k=1}^{n} a_{k} \chi_{A_{k}^{i}}$ and $Y_{i}=\sum_{k=1}^{n} a_{k} \chi_{B_{k}^{i}}(i=1,2)$ simple random variables with $\rho\left(X_{i}, Y_{i}\right)<\delta, 0<\delta<\epsilon$, if $\hat{\alpha}_{i}:[a, b] \longrightarrow L^{0}(\Omega, S)$ is the segment joining $X_{i}$ to $Y_{i}$ $(i=1,2)$, then $\rho\left(\hat{\alpha}_{1}(t), \hat{\alpha}_{2}(t)\right)<C \delta$.

We will need the following observation (a simple consequence of the definition of the metric $\rho$ ) in the proof of Lemma 4.7.

Lemma 4.8. Let $X=\sum_{i=1}^{m} a_{i} \chi_{A_{i}}$ and $Y=\sum_{i=1}^{m} a_{i} \chi_{B_{i}}$ be simple random variables, and let $\epsilon=\min \left\{d\left(a_{i}, a_{j}\right): i \neq j\right\}$. Then if $\rho(X, Y)=\delta<\epsilon$,

$$
P\left(\bigcup_{i \neq j}\left(A_{i} \cap B_{j}\right)\right)=\sum_{i \neq j} P\left(A_{i} \cap B_{j}\right) \leq \delta .
$$

Proof of Lemma 4.7. Pick $\epsilon=\min \left\{d\left(a_{i}, a_{k}\right): i \neq k\right\}$, and let $\delta<\epsilon$. We employ the notation $E_{i j}=A_{i}^{1} \cap B_{j}^{1}, E_{i j}^{\prime}=A_{i}^{2} \cap B_{j}^{2}, P\left(E_{i j}\right)=e_{i j}$, and $P\left(E_{i j}^{\prime}\right)=e_{i j}^{\prime}$. First we compute (using Definition 4.2)

$$
(\hat{\alpha}(t))^{-1}\left(a_{l}\right)=E_{l l} \cup\left(\bigcup_{k \neq l}\left[E_{k l}\right]_{t e_{k l}}\right) \cup\left(\bigcup_{k \neq l} E_{l k} \backslash\left[E_{l k}\right]_{t e_{l k}}\right),
$$

and in a similar way we can compute $(\hat{\beta}(t))^{-1}\left(a_{k}\right)$.

We must estimate $P\left((\hat{\alpha}(t))^{-1}\left(a_{l}\right) \cap(\hat{\beta}(t))^{-1}\left(a_{k}\right)\right)$, for $k \neq l$. By the choice of $\delta$, using Lemma 4.8, we can estimate

$$
\begin{gathered}
P\left(\bigcup_{k \neq p} \text { or } \bigcup_{l \neq q}\left[E_{k l}\right]_{t_{1} e_{k l}} \cap\left[E_{p q}^{\prime}\right]_{t_{2} e_{p q}^{\prime}}\right)<\delta, \\
P\left(\bigcup_{k \neq p} \text { or } \bigcup_{l \neq q}\left[E_{k l}\right]_{t e_{k l}} \cap\left(\left[E_{p q}^{\prime}\right] \backslash\left[E_{p q}^{\prime}\right]_{t_{2} e_{p q}^{\prime}}\right)\right)<\delta,
\end{gathered}
$$

and

$$
P\left(\bigcup_{k \neq p} \text { or }{ }_{l \neq q}\left(\left[E_{k l}\right] \backslash\left[E_{k l}\right]_{t e_{k l}}\right) \cap\left(E_{p q}^{\prime} \backslash\left[E_{p q}^{\prime}\right]_{t_{2} e_{p q}^{\prime}}\right)\right)<\delta .
$$

Finally, using Lemma 4.6, we estimate

$$
P\left(\left(E_{k k} \cap E_{l l}^{\prime}\right) \cup\left(\left[E_{k l}\right]_{t e_{k l}} \cap\left(E_{k l}^{\prime} \backslash\left[E_{k l}^{\prime}\right]_{t e_{k l}^{\prime}}\right)\right)\right)<\delta .
$$

This shows that $P(\{\omega: \rho(\hat{\alpha}(t)(\omega), \hat{\beta}(t)(\omega))>\delta\})<C \delta$, and $C$ only depends on the number of different values taken by the simple random variables $X_{i}, Y_{i}, i=1,2$. This completes the proof of the lemma. 
Now we are ready to prove the following.

Proposition 4.3. n-extensions are Lipschitz continuous functions (recall: the vertices are simple random variables).

Proof. Notice that 1-extensions, being segments, are Lipschitz continuous. Assume that $(n-1)$-extensions are Lipschitz continuous. Let $\hat{H}^{n}: \Pi_{k=1}^{n}\left[a_{k}, b_{k}\right] \longrightarrow$ $L^{0}(\Omega, S)$ be an $n$-extension (of course whose vertices are simple random variables). Also, it is easy to see that $\hat{H}\left(a_{1}, t_{2}, \ldots, t_{n}\right)$ and $\hat{H}\left(b_{1}, t_{2}, \ldots, t_{n}\right)$ are $(n-1)$-extensions. Let $\epsilon>0$ be given by Lemma 4.7 for $a_{1}, \ldots, a_{n}$, the values taken by the vertices of $\hat{H}^{n}$. By hypothesis, we can choose $\delta$ such that $\rho\left(\hat{H}^{n}\left(x, t_{2}^{0}, \ldots, t_{n}^{0}\right), \hat{H}^{n}\left(x, t_{2}^{0}, \ldots, t_{n}^{0}+\delta\right)\right)<C^{\prime} \delta<\epsilon, x=a_{1}, b_{1}$. Notice that for $t_{2}^{0}, \ldots, t_{n}^{0}$ fixed, $\hat{H}^{n}\left(t, t_{2}^{0}, \ldots, t_{n}^{0}\right)$ is a segment joining $\hat{H}^{n}\left(a_{1}, t_{2}^{0}, \ldots, t_{n}^{0}\right)$ and $\hat{H}^{n}\left(b_{1}, t_{2}^{0}, \ldots, t_{n}^{0}\right)$. Then, by Lemma 4.7, for any $t_{1} \in\left[a_{1}, b_{1}\right]$,

$$
\rho\left(\hat{H}^{n}\left(t_{1}, t_{2}^{0}, \ldots, t_{n}^{0}\right), \hat{H}^{n}\left(t_{1}, t_{2}^{0}, \ldots, t_{n}^{0}+\delta\right)\right)<C \delta .
$$

This shows that for $t_{1}^{0}, \ldots, t_{n-1}^{0}$ fixed, $\hat{H}^{n}\left(t_{1}^{0}, \ldots, t_{n-1}^{0}, t_{n}\right)$ is Lipschitz continuous. Since by hypothesis, for $t_{n}^{0}$ fixed, $H^{n}$ is Lipschitz continuous, the proposition follows.

We can lift $n$-polyhedrals in the obvious way. We do not have to worry about what happens at common $(n-k)$-extensions of the liftings of $n$-faces that have a common $(n-k)$-face, since by using induced families these are uniquely defined given its vertices.

Remark 3. Notice that given an $n$-extension $\hat{\alpha}^{n}: \Pi_{k=1}^{n}\left[a_{k}, b_{k}\right] \longrightarrow L^{0}(\Omega, S)$, if the distance between any two of its vertices is less than $\epsilon$, by construction and using Lemma 4.5] $\rho\left(\hat{\alpha}^{n}(\mathbf{t}), \hat{\alpha}^{n}\left(i_{1}, \ldots, i_{n}\right)\right)<n \epsilon\left(i_{k}=a_{k}, b_{k}\right)$.

Lemma 4.9. Let $H_{1}:[0,1]^{n} \rightarrow \mathcal{P}(S)$ be continuous and let $\epsilon>0$ be given. Let $\mathrm{H}_{2}$ be an arbitrary polyhedral with vertices at measures of finite support and such that

$$
\sup _{\mathbf{t} \in[0,1]^{n}} q\left(H_{1}(\mathbf{t}), H_{2}(\mathbf{t})\right)<\epsilon .
$$

Then, given any continuous lifting $\hat{H}_{1}$ of $H_{1}$, there is a continuous lifting $\hat{H}_{2}$ of $\mathrm{H}_{2}$ such that

$$
\sup _{\mathbf{t} \in[0,1]^{n}} \rho\left(\hat{H}_{1}(\mathbf{t}), \hat{H}_{2}(\mathbf{t})\right)<(3 n+2) \epsilon .
$$

Moreover (and this can be seen from the proof), we can prescribe finitely many values $X_{k}=\hat{H}_{2}\left(\mathbf{t}_{k}\right), \mathbf{t}_{k} \in \partial[0,1]^{n}$ as long as these prescribed values are simple random variables, and also

$$
\rho\left(X_{k}, \hat{H}_{1}\left(\mathbf{t}_{k}\right)\right)<\epsilon .
$$

Proof. Let $\hat{H}_{1}$ be a lifting of $H_{1}$. Take a partition $0=t_{0}<t_{1}<\cdots<t_{n-1}<$ $t_{n}=1$, such that if $\mathbf{t}, \mathbf{s} \in \Pi_{k=1}^{n}\left[t_{i_{k}}, t_{i_{k}+1}\right]$, then $\rho\left(\hat{H}_{1}(\mathbf{t}), \hat{H}_{1}(\mathbf{s})\right)<\epsilon$. Let $Y_{i_{1}, \ldots, i_{n}}$ be simple random variables such that $\operatorname{law}\left(Y_{i_{1}, \ldots, i_{n}}\right)=H_{2}\left(t_{i_{1}}, \ldots, t_{i_{n}}\right)$ and $\rho\left(Y_{i_{1}, \ldots, i_{n}}, \hat{H}_{1}\left(t_{i_{1}}, \ldots, t_{i_{n}}\right)\right)<\epsilon$. Define $\hat{H}_{2}: \Pi_{k=1}^{n}\left[a_{k}, b_{k}\right] \longrightarrow L^{0}(\Omega, S)$ as the continuous function such that $\left.\hat{H}_{2}\right|_{\Pi_{k=1}^{n}\left[t_{i_{k}}, t_{i_{k}+1}\right]}$ is the $n$-extension with vertices 
$Y_{i_{1}, \ldots, i_{n}}=\hat{H}_{2}\left(t_{1}, \ldots, t_{i_{n}}\right)$. Using the previous remark and the triangle inequality, the lemma follows.

Proof of Theorem 3. Pick partitions $0=t_{0, k}<t_{1, k}<\cdots<t_{n_{k-1}, k}<t_{n_{k}, k}=1$, where the partition of index $k+1$ is a refinement of the partition of index $k$, and such that for any $\mathbf{t}, \mathbf{s} \in \Pi_{k=1}^{n}\left[t_{i_{k}, m}, t_{i_{k}, m}\right] \cap \partial[0,1]^{n}$,

$$
\rho(X(\mathbf{t}), X(\mathbf{s}))<\frac{1}{(3 n+2)^{m+1}} .
$$

Choose simple random variables $\left(X_{m, k, i_{1}, \ldots, i_{n}}\right)_{m=1,2,3 \ldots ; 0 \leq k \leq m}$ such that

$$
\rho\left(X_{m, k, i_{1}, \ldots, i_{n}}, X\left(t_{i_{1}, k}, \ldots, t_{i_{n}, k}\right)\right)<\frac{1}{(3 n+2)^{m+1}},
$$

$\left(t_{i_{1}, k}, \ldots, t_{i_{n}, k}\right) \in \partial[0,1]^{n}$ and also

$$
\rho\left(X_{m, m, i_{1}, \ldots, i_{n}}, X_{m+1, m, i_{1}, \ldots, i_{n}}\right)<\frac{1}{(3 n+2)^{m+1}} .
$$

Since we can find a sequence of polyhedrals $\left(H_{m}\right)_{m}$, such that $H_{m} \rightarrow H$ uniformly, and $\sup _{\mathbf{t} \in[0,1]^{n}} q\left(H_{m}(\mathbf{t}), H_{m+1}(\mathbf{t})\right)<\frac{1}{(3 n+2)^{m+1}}$, by Lemma 4.9. we can construct a sequence of functions $\hat{H}_{m}:[0,1]^{n} \longrightarrow L^{0}(\Omega, S)$ such that

$\hat{H}_{m}\left(t_{i_{1}, k}, \ldots, t_{i_{n}, k}\right)=X_{m, k, i_{1}, \ldots, i_{n}}$ and $\sup _{\mathbf{t} \in[0,1]^{n}} \rho\left(\hat{H}_{m}(\mathbf{t}), \hat{H}_{m+1}(\mathbf{t})\right)<\frac{1}{(3 n+2)^{m}}$.

By all the choices made and Remark 3 we obtain

$$
\sup _{\mathbf{t} \in \partial[0,1]^{n}} \rho\left(\hat{H}_{m}(\mathbf{t}), X(\mathbf{t})\right)<\frac{n+2}{(3 n+2)^{m+1}} .
$$

From this construction it follows that $\hat{H}=\lim _{m \rightarrow \infty} \hat{H}_{m}$ is a continuous lifting of $H$ with the prescribed boundary values, i.e., $\left.\hat{H}\right|_{\partial[0,1]^{n}}=X$.

4.4. Corollary 1: Last remarks. The hypothesis of nonatomicity in Theorems 2 and 3 is necessary if $S$ is not simply connected. As pointed out to us by the referee, if $\Omega$ is a one-point probability space and $S$ is $S^{1}$, the unit circle, then $L^{0}(\Omega, S)$ is homeomorphic to $S^{1}$. On the other hand, if $S$ is a Banach space, then $L^{0}(\Omega, S)$ has a natural vector space structure compatible with the topology of convergence in probability (i.e., multiplication by scalar and sum are continuous operations), and hence it is easy to show that all its homotopy groups are trivial. However, our result shows that no matter what the topology of the Polish space $S$ is, this is always the case.

\section{Acknowledgments}

I thank the referee for carefully reading this manuscript and for many helpful comments and corrections. Also, I thank my friend Jaime Dávila for many interesting conversations about the proofs of the main results of this paper. 


\section{REFERENCES}

[BD] D. Blackwell and L. Dubins, An extension of Skorokhod's almost sure representation theorem, Proc. Amer. Math. Soc. 89 (1983), 691-692. MR718998 (86b:60005)

[V] R. de La Vega, Personal Communication.

[EK] S. Ethier and T. Kurtz, Markov Processes: Characterization and Convergence, Wiley Series in Probability and Mathematical Statistics, John Wiley and Sons, 1986. MR838085 (88a:60130)

[S] V. Strassen, The existence of probability measures with given marginals, Ann. Math. Statist. 36, 1965. MR0177430 (31:1693)

Departamento de Matemáticas, Universidad de Los Andes, Bogotá DC, Colombia

E-mail address: jean.cortissoz@gmail.com 